\title{
COMENTARIO A LA SENTENCIA DEL TRIBUNAL SUPREMO 1/2019
}

\section{Comment on the Judgment of the Supreme Court $1 / 2019$}

\author{
Fernando Costa Gilabert \\ Universidad Pontificia Comillas \\ E-mail: fercos2001@gmail.com
}

La sentencia que resuelve el recuso de casación interpuesto por el abogado del Estado en representación de la Administración General del Estado contra la decisión de la Audiencia Nacional, por la que se procedía a anular una resolución sancionadora de la CNC, desestima el mismo y, a su vez, sienta doctrina acerca dos cuestiones. La primera consiste en determinar el alcance y extensión del control jurisdiccional sobre las apreciaciones económicas complejas que puede llevar a cabo un órgano regulador de un sector haciendo uso del margen de apreciación de que dispone en base al ámbito de sus competencias; la segunda, en determinar si la delimitación del mercado relevante que había hecho la CNC para sancionar a Vodafone podía vulnerar el art. 2 LDC y la jurisprudencia comunitaria.

The judgment that resolved the appeal filed by the State Attorney on behalf of the General State Administration against the decision of the Audiencia Nacional which resulted in the annulation of a sanctioning resolution of the CNC, resulted in the appeal's dismissal and doctrine's establishment on two important issues. The first one consists of determining the scope and extent of judicial control over complex economic appreciations that are carried out by the regulator of a sector, making use of the margin of appreciation available based on its powers; the second, to determine whether the delimitation of the relevant market that the CNC had made to sanction Vodafone could or not violate art. 2 LDC and the Community case law.

Mercado relevante; posición de dominio; abuso de posición de dominio; margen de apreciación; control judicial; recurso de casación; competencia

Key words

Relevant market; position of dominance; abuse of position of dominance; margin of appreciation; judicial control; appeal; competition

\section{Resumen}




\section{Introducción}

En este trabajo procedemos a comentar la sentencia del TS 1/2019 de 8 de enero, encargada de la resolución del recurso de casación interpuesto por el abogado del Estado contra la sentencia de la Audiencia Nacional 3555/2017 del 1 de septiembre. En esta sentencia de la AN se estima el recurso contencioso-administrativo interpuesto por Vodafone frente a la resolución sancionadora de la Comisión Nacional de la Competencia (expediente sancionador S/0248/10 Mensajes Cortos) que hacía responsable a Vodafone (junto con Telefónica y France-Telecom) de un abuso de posición de dominio en el mercado de servicios mayoristas de terminación de mensajes cortos y a su vez imponía una multa de 43252000 euros, quedando con esta sentencia anulada, pero siendo susceptible del recurso que en este comentario procedemos a analizar en profundidad, habiendo considerado pertinente también, a efectos de ponernos en contexto, una pequeña explicación de cómo ha evolucionado el sector de las telecomunicaciones en los últimos tiempos.

\section{Evolución del sector}

Como bien se manifiesta tanto en la sentencia de la Audiencia Nacional como en la del TS, el sector de las telecomunicaciones es increíblemente dinámico, habiendo experimentado una grandísima evolución desde su nacimiento. Efectivamente, el mercado de telefonía en España tiene su origen en 1976 en el que actuaba Telefónica (empresa pública en dicho momento) en régimen de monopolio, pudiendo afirmar así que, en dicho momento, la prestación de servicios en el sector de las telecomunicaciones consistía en un servicio público en sentido estricto (consistía en un monopolio natural, organizado alrededor de una red que explotaba directamente el Estado).

Esto cambia en gran medida con la normativa de la UE (principalmente directivas) por la que se determinaba la apertura de numerosos sectores a la competencia permitiendo la entrada de nuevos operadores, y que materializa precisamente cuando se produce el ingreso de Vodafone en 1995 en este sector de las telecomunicaciones con su red propia, pasando así el mismo a ser un servicio público en sentido impropio (ya no se da el monopolio de la administración en la prestación de servicios, pues hay concurrencia en la prestación con el sector privado). Este sector continúa creciendo, llegando diferentes empresas que conviene distinguir: por un lado, las OMR (aquellas empresas que cuentan con una red propia para prestar sus servicios) y, por otro, las OMV (aquellas sin red propia pero que alquilan la red de los OMR para prestar sus servicios).

En este contexto se produce la resolución de la Comisión del Mercado de las Telecomunicaciones del 2 de febrero de 2006, por la que se impone a los operadores con red propia (OMR) la obligación de dar acceso a sus redes al resto de los operadores a cambio de un precio (obligación de servicio público), por lo que la terminación de mensajes en la red de otro operador supondría el pago al operador de la red ("si usas pagas"), pudiendo considerarse así el sector de las telecomunicaciones un servicio de interés económico general, lo cual se manifiesta en el hecho de que se intente hacer compatible la liberalización del sector con la garantía de que se presten los servicios esenciales en el mismo, y para ello se impone esa obligación de servicio público a los operadores con red propia (si no se impusiera podrían seguir manteniéndose los monopolios o sería sencillo que se llevasen a cabo prácticas discriminatorias), y su financiación se lleva a cabo a través de un fondo común en el sector. 
De esta forma apreciamos cómo en el sector de las telecomunicaciones se ha pasado de actuar solamente un operador en régimen de monopolio, a la existencia de un gran numero de estos que aseguran una gran competencia en el sector, teniendo en cuenta las obligaciones se servicio público que tienen los operadores con red propia respecto de los que no.

\section{Descripción del caso}

\subsection{Sentencia de la AN}

En virtud del recurso interpuesto por Vodafone contra la resolución sancionadora de la CNC por la que se imputa a Vodafone el abuso de la posición de dominio en el mercado mayorista de la terminación de mensajes por la fijación de precios excesivos, se dicta esta sentencia de la AN que procede a anular el acto del ente regulador del sector. Esta resolución sostenía que no solo existía un mercado separado en la terminación de mensajes directos, sino que además existía un mercado diferente en cada red propia, teniendo los operadores de dichas redes el $100 \%$ de la cuota de ese mercado separado. En nuestro análisis nos centraremos en los Fundamentos de Derecho por los que la AN llega a la conclusión de que se ha de anular la misma.

- Lo primero que debe hacer la CNC para imputar a Vodafone un abuso de posición de dominio es definir el mercado relevante en el que se produce con la suficiente motivación e investigación.

- En segundo lugar, debe probar que existe posición de dominio en dicho mercado, que se manifestaría en una posible actuación independiente de Vodafone en el mismo.

- El abuso de la posición de dominio se correspondería con una actuación que restringiese la competencia en el sector, expulsando a los competidores o tratando de privar al consumidor de poder elegir con quien quiere contratar.

- La CNC en el empleo de apreciaciones económicas complejas para determinar si las condiciones anteriores se presentaban o no, se encuentra bajo control judicial de acuerdo a la jurisprudencia comunitaria, que se encarga de verificar la racionalidad de la decisión de la autoridad de la competencia a la vista de los datos tomados en consideración para alcanzar la decisión, y la exactitud de los elementos probatorios junto con su fiabilidad y coherencia.

La AN considera en primer lugar, con respecto a la delimitación del mercado relevante exclusivo para la terminación de mensajes, que no se da una motivación y un análisis propio de la CNC que pueda permitir la consideración del mismo, debido a que para ello hace uso de un informe de la CMT que tenía como finalidad una regulación ex ante del mercado de llamadas y que se traslada sin justificación al terreno de la actuación represiva por infracción de derecho de la competencia en el mercado de terminación de mensajes (ha de ser un análisis de mercado diferente el enfocado a una regulación ex ante al de una posible represión de una conducta, porque las conclusiones que se extraerán de los mismos pueden diferir en virtud del fin del informe). A su vez destaca la incoherencia manifestada por la CNC al apoyarse para determinar la existencia del mercado separado de SMS en un informe (mercado 16) en el que se manifiesta que no era lógica una regulación ex ante del mercado de comercialización 
de SMS, dando a entender que no es necesario por no existir problemas de competencia en el mismo. Además, se destaca a su vez el hecho de que se archivase una posible colusión de los principales operadores del sector (Vodafone, Telefónica, Orange...) en el mismo tiempo en el que la CNC sostiene que se dio el abuso de la posición dominante. Con respecto a la delimitación de un mercado para cada red, de nuevo la AN considera que no existe una suficiente explicación y análisis que lleven a alcanzar esta conclusión, siendo por tanto inconsistente. A estas conclusiones llega el tribunal por entenderse competente para controlar judicialmente las apreciaciones económicas complejas que lleva a cabo la autoridad competente en virtud de la jurisprudencia comunitaria.

Pese a ello, la AN en segundo lugar plantea la hipótesis de que se hubiese aceptado la delimitación de mercado de la $\mathrm{CNC}$, procediendo así a analizar si existe posible posición de dominio. Cuando lleva a cabo el examen para analizarlo, llega a la conclusión de que no se produce, debido a que posición de dominio de una empresa implica el que esta pueda actuar de forma independiente en el mercado relevante con respecto al resto de operadores, mientras que en el mercado de terminación de mensajes lo que se da es un monopolio bilateral, por lo que existe una interdependencia con respecto al resto de operadores que implica la necesidad de acordar términos y precios a la hora de emplear las redes de otros operadores. Con respecto a la afirmación que hace la CNC en su resolución sancionadora de que las OMV y Yoigo no están en las mismas condiciones a la hora de negociar esos precios de acceso a la red de las OMR, existiendo por tanto una posición de asimetría y sin suficiente poder de compensación, un dictamen pericial aportado por Vodafone sostiene que no se ha planteado en ningún momento por parte de las OMV un conflicto de interconexión en atención a peticiones de precios equitativos (a su vez no se entiende cómo, si se está abusando de estos operadores, la cuota de mercado de Yoigo haya estado creciendo durante el periodo en el que supuestamente se da la infracción), y al mismo tiempo que la fijación de precios altos por parte de Vodafone podría suponer un perjuicio para ella, debido a que envía a otras redes de operadores más mensajes de los que recibe.

La AN estima que, al no existir posición de dominio, no es necesario analizar un posible abuso, y procede a estimar en su sentencia el recurso de Vodafone por el que se anula el acto, pero cabiendo contra la misma recurso de casación.

\subsection{Antecedentes de hechos del TS}

Como expusimos supra, la sentencia de la AN es recurrida en casación por la AGE que se encuentra representada y defendida por el abogado del Estado. El recurso es estimado y se manifiesta en el auto de admisión la existencia de dos puntos que presentan interés casacional consistentes:

Esclarecer la extensión y límites del control judicial a las resoluciones de la CNMC que impliquen apreciaciones económicas complejas (especialmente en la delimitación de mercados para determinar una posible posición dominante y una actuación abusiva consecuencia de la anterior).

Apreciar si se vulnera el art. 2 LDC y la jurisprudencia comunitaria de la Sentencia de la Audiencia Nacional por una delimitación del mercado de referencia para cada uno de los operadores Telefónica, Vodafone y Orange, consistente en el mercado mayorista de terminación 
de mensajes integrado exclusivamente por la propia red de cada operador (así, cada operador tiene el 100\% de cuota en el mercado que integra su red).

El abogado del Estado en el recurso solicitaba una interpretación de la normativa y de la jurisprudencia comunitaria por la que se anulase la sentencia dictada en instancia.

\section{Posición de las partes en el TS}

\subsection{AGE a través del abogado del Estado}

El abogado del Estado mantiene que ha tenido lugar una infracción en las reglas de motivación y exhaustividad alegando para ello la STJUE asunto C-12/03 P (en la que la Comisión sostiene que el Tribunal de la UE se excedió en sus competencias de control), y también destaca la falta de racionalidad y lógica en la sentencia recurrida, argumentando a su vez la infracción de la jurisprudencia comunitaria.

En primer lugar, sostiene que no se puede acudir a diferentes sentencias del TJUE para anular la resolución de la $\mathrm{CNC}$ por falta de motivación cuando no se han explicado ni los motivos por los que no se puede acudir a informes previos como el de la CMT para delimitar un mercado relevante, ni tampoco cuáles son los datos que han de tomarse en consideración a la hora de llevar a cabo dicha determinación de mercado (en definitiva, plantea qué debe emplearse para delimitar un sector).

Por otro lado, cuestiona el ámbito al que se extiende el control judicial sobre las apreciaciones económicas complejas que hace la autoridad de la competencia en virtud de su margen de apreciación.

\subsection{Vodafone}

La posición de Vodafone se recoge, como manifiesta el auto de admisión del recurso de casación, en los argumentos que esta ofreció en la Audiencia Nacional sobre la falta de delimitación del mercado de referencia, y por los que esta consideró que efectivamente no se había llevado a cabo un análisis propio para el caso concreto, sino que se había fundado en informes previos de la CMT referidos a la necesidad de una regulación ex ante del mercado de llamadas e igual para la delimitación de un mercado para cada red de operadores. Aparecen estos argumentos en la sentencia de la Audiencia Nacional como los motivos por los que Vodafone solicita la anulación del acto, siendo los más importantes a nuestro parecer:

Non bisin idem: como expusimos anteriormente, una resolución de 2008 archivó una denuncia de posible colusión, pero ahora se castiga una conducta abusiva (contradicción de la CNC) en relación con una fijación de precios elevada cuando existen obligaciones de servicio que desincentivan el prevalecimiento de una posible posición de dominio existiendo competitividad y poder compensatorio por las OMV, y además se reconoce que en el sector existen monopolios bilaterales.

Desviación de poder: la CNC se excede en sus poderes al establecer una sanción que tiene por fin reestructurar un sector cuando ello es competencia de la CMT. 
Atipicidad en la conducta: no se puede afirmar ni que hay un abuso de posición de dominio cuando la propia estructura de mercado al estar constituida en monopolios bilaterales lo impide porque no se pueden adoptar comportamientos independientes, ni tampoco que se han fijado precios altos cuando este viene condicionado por los monopolios bilaterales, las obligaciones de interconexión, las características propias del sector y la influencia de la CMT (se ha de comparar el precio con los costes que supone una prestación global de servicios, porque solo el de la terminación de mensajes no es rentable económicamente).

Inexistencia de abuso: al no existir posición dominante no se puede abusar de la misma.

Inexistencia de culpa: no se podía prever la sanción y se siguieron en todo caso las indicaciones de la CMT.

\section{Decisión del Tribunal Supremo}

\subsection{Fundamentos de la decisión del TS y decisión (jurisprudencia tomada en consideración)}

El examen de este recurso de casación se llevó a cabo al mismo tiempo que el de los planteados por el abogado del Estado contra las sentencias de la Audiencia Nacional relativas a Telefónica y a Orange (France-Telecom), existiendo en todos ellos unos argumentos similares. Por esta razón es de vital importancia la sentencia del Tribunal Supremo que resuelve el recurso de casación contra la sentencia de la Audiencia Nacional de Telefónica en la que destacamos los fundamentos de derecho 2.o, 3.o y 4...:

En el fundamento de derecho 2.o de esta sentencia se resuelve acerca del control judicial de las apreciaciones económicas complejas que lleva a cabo el ente regulador (se resuelve la cuestión de interés casacional relativa a la extensión y límites del control judicial sobre las apreciaciones de esta naturaleza de la CNMC). En este fundamento se reconoce la existencia de abundante jurisprudencia comunitaria que pone de manifiesto que el alcance del control judicial a las apreciaciones económicas de la comisión se extiende a verificar la racionalidad de la conclusión a la que esta llega haciendo uso de las mismas, pudiendo trasladarse esta doctrina perfectamente a los tribunales españoles (dentro de las apreciaciones que se someten a dicho control figuran aquellas destinadas a la delimitación de un mercado relevante). De esta manera, el control judicial comprende la exactitud de los elementos probatorios, la fiabilidad y coherencia de estos, así como la pertinencia y adecuación de los datos empleados para alcanzar una conclusión (en caso de entender que no es coherente puede anular el acto). Por esta doctrina asentada en la jurisprudencia comunitaria en sentencias del TJUE como la de Coca-Cola, Tetra Laval o Schneider/Legrand, el tribunal de instancia analizó los datos e informes en los que se basó la resolución de la $\mathrm{CNC}$ para delimitar el mercado relevante y posteriormente analizar una posible posición de dominio y concluyó que los datos empleados eran insuficientes e incoherentes, procediendo la anulación de la misma.

El fundamento de derecho 3.o se encargaba de analizar el alcance del control casacional (resuelve la segunda cuestión que planteaba interés casacional, sobre la posible vulneración del art. 2 LDC y de la jurisprudencia comunitaria por la delimitación del mercado de referencia para cada operador consistente en un mercado de terminación de mensajes cortos integrado solo por la propia red del operador, teniendo este así un 100\% de cuota de mercado). En es- 
te fundamento el tribunal reconoce que sus facultades de casación se circunscriben no a delimitar el mercado relevante ni a controlar la suficiencia probatoria de la CNMC al definir un mercado relevante y las posibles posiciones de dominio en el mismo, sino que evalúa si el tribunal de instancia cumplió con los requisitos fijados por la jurisprudencia al examinar si la información empleada por el ente regulador para la delimitación del mercado era suficiente para avalar la conclusión a la que llega de que existe un mercado separado de mensajes cortos y que la posición de los operadores en el mismo venía dado en relación a sus propias redes. El TS se limita a analizar cuestiones de derecho sin entrar en las de hecho ni en la valoración de pruebas, y como el recurso interpuesto lo que pretendía era una nueva valoración de los datos, no es algo que le corresponde al TS, sino que ya llevó a cabo la AN.

El 4.o fundamento de derecho resuelve acerca de la necesidad de elaborar el ente regulador del sector un análisis propio para delimitar el mercado relevante. Como bien sabemos, la AN consideró que no podía basarse la $\mathrm{CNC}$ en la delimitación del mercado relevante para destacar una posición de dominio y un eventual abuso en previsiones anteriores y en relación con un mercado distinto (el informe de la CMT era relativo a la necesidad de una regulación en el mercado de las llamadas), y el recurso que se resuelve cuestionaba esta afirmación sosteniendo que la valoración que se había llevado a cabo del soporte empleado por la CNC es erróneo junto con una aplicación incorrecta de la jurisprudencia comunitaria en relación a la extensión del control judicial sobre las apreciaciones económicas y que el organismo regulador puede delimitar el mercado relevante con informes previos (si están actualizados y son coetáneos) sin ser preciso un informe propio.

Frente a la alegación de la aplicación incorrecta de la jurisprudencia comunitaria, el TS mantiene que en abundante jurisprudencia comunitaria, concretamente en la STJUE Schindler, se reconoce el extenso control judicial sobre las apreciaciones del ente regulador, algo que se acompaña de sentencias como la de Tetra Laval o Chalkor, en las que se afirma que el TJUE no puede renunciar a sus competencias de control en cuestiones de hecho y de derecho alegando el margen de apreciación de la comisión, disponiendo por tanto de amplias facultades que van desde la valoración de las pruebas, a la anulación de decisiones y modificación de multas.

Con respecto al deber de análisis propio del mercado relevante, se destaca la STJUE Continental Can en la que se afirma la vital trascendencia de la delimitación del mercado relevante (productos no son intercambiables) y la STJUE Hoffman-LaRoche, en la que se recalca que la intercambiabilidad de los productos a la hora de delimitarse un mercado ha de apreciarse junto con las características objetivas de los productos y servicios relevantes, las condiciones de competencia y de oferta y demanda en el mercado (STJUE Michelín). En virtud de estas sentencias se aprecia la vital importancia de delimitar de forma precisa el mercado relevante, algo que no supone que no se puedan emplear informes previos en ello (siempre que se encuentren actualizados), pero tampoco dispensa de la obligación de llevar a cabo un análisis propio del mercado por parte del ente regulador cuando va a hacer uso de su facultad sancionadora, algo que se manifiesta en la STJUE Coca-Cola, por lo que no se puede apoyar la delimitación del mercado en un informe previo relativo a un mercado distinto. Esto se debe a que las conclusiones que se extraen de los exámenes relativos a la determinación de mercados relevantes son diferentes cuando estos tienen una finalidad prospectiva a cuando por el contrario es represiva. El problema es que en nuestro caso, no solo hay una ausencia de análisis propio, sino que además existen contradicciones en el informe empleado para la delimitación del mercado (se prevé que no es necesario una regulación ex ante para el mercado de comercialización de SMS por existir una alta competitividad) e incluso en las conclusiones que de este derivan e incohe- 
rencias con otras resoluciones (se apoya la existencia del mercado de terminación de mensajes en la necesidad de regulación del mercado de llamadas).

Por todo ello, en la resolución del recurso de casación de Telefónica se procede a mantener la anulación de la resolución sancionadora de la CNC por datos insuficientes y no objetivos, incoherencia y no motivación en la delimitación del mercado y en la determinación de las posiciones de dominio de los operadores.

\subsection{Doctrina asentada por el TS}

El TS con este recurso de casación establece doctrina en relación a las dos cuestiones que planteaban interés casacional (la misma que la de la sentencia que resuelve el recurso de casación de Telefónica):

Con respecto a la primera relativa a los límites del control judicial de las apreciaciones de la CNMC, sostiene que esta se extiende hasta decidir sobre la racionalidad de la decisión, pudiendo incluso llegar a anularla si entiende que no.

En relación a la segunda, fundada en si se había o no vulnerado el art. 2 LDC y la jurisprudencia comunitaria por la delimitación de un mercado de relevante en el mercado mayorista de terminación de mensajes para cada operador con red propia, considera que no corresponde al TS establecer el mercado relevante ni el control de la suficiente prueba por parte de la CNMC para definirlo o reconocer la posición de dominio de una empresa, sino que corresponde al tribunal de instancia apreciar si la información empleada para ello es o no suficiente. Se reconoce que el TS solo analiza las cuestiones de derecho y que su función es apreciar si el tribunal de instancia ejerció su control de forma correcta. Por último, se destaca que la determinación del mercado relevante puede hacerse en base a numerosos datos por el ente regulador, pero nunca ello dispensa de la obligación de análisis propio basado en todos los datos adecuados y disponibles al ejercer la potestad sancionadora.

\subsection{Fallo}

El TS a la vista de todo lo expuesto procede a desestimar el recurso de casación interpuesto contra la sentencia de la AN.

\section{Conclusiones}

En el análisis sobre la sentencia del TS hemos apreciado diferentes cuestiones como la regulación en los mercados (establecimiento reglas del juego) y la posterior supervisión (cumplimiento de las reglas) que se da en los mismos por parte de las administraciones. Hemos apreciado una posible vulneración del derecho de la competencia por un abuso de posición dominante, con las facultades de inspección y sanción que tienen los entes reguladores de los sectores para luchar contra ello (también el problema que existía antes de la CNMC en los sectores regulados, al encargarse la $\mathrm{CNC}$ de aplicar la normativa horizontal del derecho de la competencia y otros órganos como la CMT de la aplicación de la normativa sectorial, con los desajustes que ello suponía. La evolución del sector ha sido clave para identificar cómo se pasa de un servicio público en sentido estricto a un servicio público en sentido impropio, y hoy en día a lo conocido como servicio de interés económico general. Por último, se ha puesto de mani- 
fiesto la existencia de entes independientes (que conforman la administración territorial) con funciones propias de supervisión de sectores y de su control judicial.

Me gustaría recalcar la enorme complejidad que existe en estos sectores en los que destacamos una regulación concreta por las circunstancias técnicas o peculiares que los definen, y la totalmente necesaria existencia de entes independientes de la coyuntura política que se encarguen de velar por un correcto funcionamiento de los mismos. Si no fuera por ellos, se daría lugar a prácticas con resultados negativos en la espera patrimonial de los consumidores sin que ellos se llegasen siquiera a dar cuenta de lo que está sucediendo, encontrándose el contrapeso de una posible intervención excesiva en la economía en el control judicial que se extiende sobre los mismos como se manifiesta en la sentencia comentada. Resulta inconcebible por razones de protección a las partes más débiles en las relaciones jurídicas en las que existe asimetría, y de interés superior como asegurar la supervivencia del sistema sin que quiebre este, una economía en la que el Estado no se encargue de establecer unas reglas mínimas de juego y una supervisión de que las mismas se cumplen.

\section{Referencias}

STS $1 / 2019$ de 8 de enero de 2019.

SAN 3555/2017 de 1 de septiembre de 2017.

Burzaco Samper, M., Lavilla Rubira, J. J., Magide Herrero, M. y Vega Labella, J. I. (2020). Derecho administrativo económico. Madrid: Dykinson. 